\title{
QPOs expected in rotating accretion flows around a supermassive black hole
}

\author{
T. Okuda, ${ }^{1}$ V. Teresi ${ }^{2}$ and D. Molteni ${ }^{2}$ \\ ${ }^{1}$ Hokkaido University of Education, Hachiman-Cho 1-2, 040-0429 Hakodate, Japan \\ ${ }^{2}$ Dipartimento di Fisica e Tecnologie Relative, Università di Palermo, Viale delle Scienza, \\ 90128 Palermo, Italy \\ email: okuda@cc.hokkyodai.ac.jp, molteni@unipa.it
}

\begin{abstract}
It is well known that rotating inviscid accretion flows with adequate injection parameters around black holes could form shock waves close to the black holes, after the flow passes through the outer sonic point and can be virtually stopped by the centrifugal force. We numerically examine such shock waves in $2 \mathrm{D}$ accretion flows with $10^{-5}$ to $10^{6}$ Eddington critical accretion rates around a supermassive black hole with $10^{6} M_{\odot}$. As the results, the luminosities show QPO phenomena with modulations of a factor 2-3 and with quasi-periods of a few to several hours.
\end{abstract}

Keywords. Accretion, accretion disks - black hole physics - galaxies: active - hydrodynamics - radiation mechanisms: thermal - shock waves

\section{Introduction}

Since the pioneer works of transonic problems of accretion and wind by Fukue(1987) and Chakrabarti and his collaborators (Chakrabarti 1989, Abramowicz \& Chakrabarti 1990, and Chakrabarti \& Molteni 1993), it has been shown that these generalized accretion flows could be responsible for the quasi-periodic oscillation (QPO) from the black hole candidates (Molteni et al. 1996.; Ryu et al. 1997.; Lanzafame et al. 1998.). Here, following the recent numerical 2D simulations of the shocks (Okuda et al. 2004.; Chakrabarti et al. 2004.), we examine the QPOs phenomena due to the centrifugally supported shocks around a supermassive black hole with $10^{6} M_{\odot}$, while taking account of the cooling and heating of the gas and the radiation transport. .

\section{Model Parameters and Numerical Methods}

For the central black hole, we consider a supermassive black hole with $10^{6} M_{\odot}$. The basic equations and the numerical methods used here are given in Okuda et al.(2004). A typical set of injection parameters, such as the specific angular momentum, $\lambda_{\text {out }}$, the radial velocity $v_{\text {out }}$, the sound velocity $a_{\text {out }}$, the ambient density $\rho_{\text {out }}$, and the accretion rate $\dot{m}$ normalized to the Eddington critical accretion rate $\dot{M}_{\mathrm{E}}\left(=2.7 \times 10^{23} \mathrm{~g} \mathrm{~s}^{-1}\right)$ at an outer boundary radius $R_{\text {out }}$, are given in table 1 . Here, the velocities and distances are

Table 1. Injection flow parameters

\begin{tabular}{lcccccc}
\hline$\lambda_{\text {out }}$ & $v_{\text {out }}$ & $a_{\text {out }}$ & $\rho_{\text {out }}\left(\mathrm{g} \mathrm{cm}^{-3}\right)$ & $\dot{m}$ & $\phi$ & $R_{\text {out }} / R_{\mathrm{g}}$ \\
\hline 1.875 & 0.0751 & 0.0654 & $10^{-17}-10^{-6}$ & $10^{-5}-10^{6}$ & $29^{\circ}$ & 30.0 \\
\hline
\end{tabular}


given in units of the speed of light, $c$, and the Schwarzschild radius, $R_{\mathrm{g}}$, respectively. $\phi$ is the subtended angle of the central black hole to the initial disk at $r=R_{\text {out }}$, that is, $\tan \phi=(h / r)_{\text {out }}$, where $h$ is the inflow thickness.

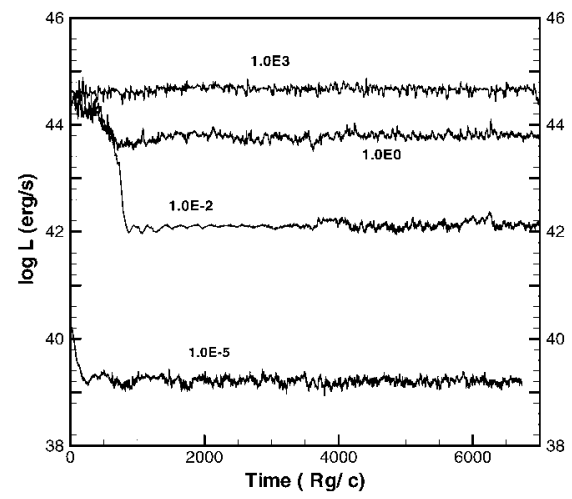

Figure 1. Luminosity $L$ as a function of time in units of $R_{\mathrm{g}} / c$ for cases of $\dot{m}=10^{-5}, 10^{-2}$, 1 , and $10^{3}$.
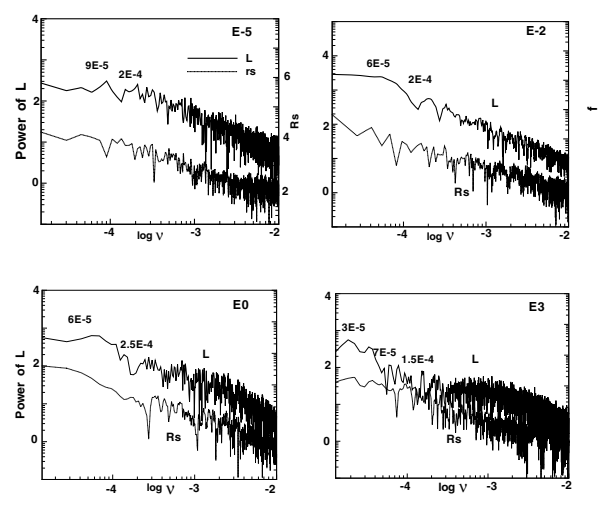

Figure 2. Power density spectra of luminosity $L$ (solid line) and shock positions $R_{\mathrm{s}}$ (dotted line) for cases of figure 1.

\section{Results}

In all of the above models, the centrifugally supported shocks are formed at the region of $5-12 R_{\mathrm{g}}$ around the black hole, depending weakly on the accretion rate, and oscillate quasi-periodically around the shock position. Figure 1 shows the luminosity curves for cases of $\dot{m}=10^{-5}, 10^{-2}, 1$, and $10^{3}$, respectively. The luminosity increases in proportion to the accretion rate when it is low, but it tends to a saturated value of $\sim 3$ Eddington luminosity when it exceeds considerably the Eddington critical rate. The power density spectra for the luminosity and the shock position on the equatorial plane are given in figure 2. Here we find the QPO frequency $\nu_{\mathrm{qpo}} \sim 3 \times 10^{-5}-3 \times 10^{-4}$, that is, the period $P \sim 1-10$ hours. The luminosities show QPO behaviour with modulations of a factor $2-3$ and with quasi-periods of a few to several hours. These results may suggest the existence of QPOs with the time scale of hours in AGNs .

\section{References}

Fukue, J. 1987, PASJ, 39, 309

Chakrabarti, S. K. 1989, ApJ, 347, 365

Abramowicz, M. A. \& Chakrabarti, S. K. 1990, ApJ, 350, 281

Chakrabarti, S. K. \& Molteni, D. 1993, ApJ, 417, 671

Molteni, D., Sponholz, H. \& Chakrabati, S. K. 1996, ApJ, 457, 805

Ryu, D., Chakrabarti, S. K. \& Molteni, D. 1997, ApJ, 474, 378

Lanzafame, G., Molteni, D. \& Chakrabarti, S. K. 1998,MNRAS, 299, 799

Okuda, T., Teresi, V. \& Molteni, D. 2004, PASJ, 56, 547

Chakrabarti, S. K., Acharyya, K. \& Molteni, D. 2004, A\&A, 421, 1 